\title{
Abordagem estética da região palpebral inferior - Uma revisão das principais opções
}

\section{terapêuticas}

\author{
Esthetic approach to the lower eyelid - A review of the main therapeutic options \\ Enfoque estético de la región del párpado inferior - Una revisión de las principales opciones
}

terapéuticas

Recebido: 13/04/2021 | Revisado: 20/04/2021 | Aceito: 21/04/2021 | Publicado: 07/05/2021

\author{
Gilmasa Daniele Rios Dias \\ ORCID: https://orcid.org/0000-0002-0284-0359 \\ Universidade de São Paulo, Brasil \\ E-mail: danielerios.oftalmo@gmail.com \\ André Borba \\ ORCID: https://orcid.org/0000-0002-2293-1119 \\ Universidade de São Paulo, Brasil \\ E-mail: prof.dr.andreborba@gmail.com
}

\begin{abstract}
Resumo
Introdução: As alterações do tear through e da região infraorbital são um dos primeiros sinais reconhecíveis de envelhecimento. Estas são queixas comuns comum na medicina estética e há muitas opções de tratamento disponíveis. Tendo isso em vista, o objetivo do presente estudo é realizar uma revisão de literatura para descrever a anatomia e as alterações da região infraorbital decorrentes do envelhecimento e apresentar as principais opções terapêuticas para cada caso. Materiais e métodos: Uma revisão simples de literatura foi realizada nas bases de dados: Pubmed, Scielo, Web of Sciences, usando os descritores: Tear trough , Infra-orbital, Dark-circles, Lower-Eyelid, Hyperpigmentation e Midface, treatment, therapeutics com seus termos correspondentes em inglês e português. Resultados: Preenchimentos, bioestimuladores de colágeno, lasers, dispositivos de radiofrequência, microagulhamento, peelings químicos, cosmecêuticos e toxina botulínica são opções de tratamento não cirúrgico disponíveis discutidas na literatura, constatou-se que melhora e rejuvenesce significativamente a região infraorbital. Conclusão: A compreensão completa da anatomia e das alterações que ocorrem com o envelhecimento são de extrema importância na avaliação da região infraorbital para determinar as opções de tratamento adequadas como uma forma de alcançar um rejuvenescimento ideal.
\end{abstract}

Palavras-chave: Tear trough; Abordagem estética; Pálpebra inferior.

\begin{abstract}
Introduction: Changes in the tear through and infraorbital region are one of the first recognizable signs of aging. These are common complaints in aesthetic medicine and there are many treatment options available. With this in mind, the purpose of this study is to perform a literature review to describe the anatomy and changes in the infraorbital region due to aging and present the main therapeutic options for each case. Materials and methods: A simple literature review was performed in the databases: Pubmed, Scielo, Web of Sciences, using the descriptors: Tear trough, Infraorbital, Dark-circles, Lower-Eyelid, Hyperpigmentation and Midface, treatment, therapeutics with their corresponding terms in English and Portuguese. Results: Fillers, collagen biostimulators, lasers, radiofrequency devices, microagulants, chemical peels, cosmeceuticals and botulinum toxin are available non-surgical treatment options discussed in the literature, it was found to significantly improve and rejuvenate the infraorbital region. Conclusion: A thorough understanding of the anatomy and changes that occur with aging are of utmost importance in evaluating the infraorbital region to determine appropriate treatment options as a way to achieve optimal rejuvenation.
\end{abstract}

Keywords: Tear trough; Aesthetic approach; Lower eyelid.

\section{Resumen}

Introducción: Changes in the tear through and infraorbital region are one of the first recognizable signs of aging. These are common complaints in aesthetic medicine and there are many treatment options available. With this in mind, the purpose of this study is to perform a literature review to describe the anatomy and changes in the infraorbital region due to aging and present the main therapeutic options for each case. Materials and methods: A simple literature review was performed in the databases: Pubmed, Scielo, Web of Sciences, using the descriptors: Tear trough, Infraorbital, Dark-circles, Lower-Eyelid, Hyperpigmentation and Midface, treatment, therapeutics with their corresponding terms in English and Portuguese. Results: Fillers, collagen biostimulators, lasers, radiofrequency devices, microagulants, chemical peels, cosmeceuticals and botulinum toxin are available non-surgical treatment 
options discussed in the literature, it was found to significantly improve and rejuvenate the infraorbital region. Conclusion: A thorough understanding of the anatomy and changes that occur with aging are of utmost importance in evaluating the infraorbital region to determine appropriate treatment options as a way to achieve optimal rejuvenation.

Palabras clave: Tear trough; Enfoque estético; Párpado inferior.

\section{Introdução}

A arte do rejuvenescimento facial mudou drasticamente na última década, evoluindo do tratamento isolado de rítides superficiais para uma abordagem que se concentra na revolumização, em grande parte devido a um entendimento mais amplo e completo das mudanças ósseas e das partes moles que ocorrem no processo de envelhecimento facial. Nos últimos anos, preenchimentos injetáveis tornaram-se parte integrante da terapia cosmética, mas eles também progrediram, tornando-se um produto durável, mas não permanente, bem tolerado, fornecendo resultados que são sutis, naturais e potencialmente reversíveis no caso de eventos clínicos desfavoráveis (J. H. Lee \& Hong, 2018; Peng \& Peng, 2018).

A região periorbital é a primeira área da face a mostrar sinais de envelhecimento. Para fornecer rejuvenescimento seguro e com aparência natural das pálpebras delicadas e das estruturas de suporte, um conhecimento avançado da anatomia, proporções faciais ideais e os métodos mais eficazes de rejuvenescimento são discutidos. O rejuvenescimento periocular é particularmente desafiador devido à intrincada e delicada anatomia da área periocular. Para garantir resultados seguros e bemsucedidos, é crucial que seja usada uma abordagem global ao fornecer tratamentos e que seja considerada uma avaliação dos tecidos moles, da vascularização e da estrutura óssea da região periocular, antes de administrar tratamentos para rejuvenescimento estético (Shah-Desai \& Joganathan, 2020).

As olheiras são uma queixa muito frequente e o preenchimento das pálpebras inferiores constitui um grande desafio, visto que as camadas teciduais são muito finas, sua anatomia é complexa e ainda há uma grande dificuldade na escolha do preenchedor adequado, assim como o plano de aplicação. As olheiras continuam difíceis de tratar, tanto cirurgicamente quanto não cirurgicamente, devido à sua natureza multifatorial (Shah-Desai \& Joganathan, 2020).

O objetivo do presente estudo é realizar uma revisão da literatura para descrever a anatomia e as alterações da região infraorbital decorrentes do envelhecimento e apresentar as diferentes opções de tratamentos da região periocular, discutindo como essas terapêuticas podem ser usadas para o rejuvenescimento ideal desta área.

\section{Metodologia}

Trata-se de um estudo do tipo qualitativo, ou seja, aqueles nos quais é importante a interpretação por parte do pesquisador com suas opiniões sobre o fenômeno em estudo, o estudo de Pereira et al. (2018) forneceu o suporte metodológico para o presente estudo. Neste foi realizada uma revisão de estudos sobre anatomia da região infraorbitária associado aos inúmeros tratamentos propostos e artigos de revisão da literatura, nas bases de dados Pubmed, Scielo e Web of Sciences, utilizando como busca, palavras-chave: Tear trough, Infra-orbital, Dark-circles, Lower-Eyelid, Hyperpigmentation e Midface,, com seus termos correspondentes em português Os dados coletados foram apresentados de forma descritiva.

Foram excluídos artigos sobre complicações e tratamento de outras áreas da face. Uma revisão abrangente da literatura foi realizada, incluindo estudos de casos, randomizados, coortes prospectivas e retrospectivas bem como artigos de revisão, no período de 1985 a 2021. Esta pesquisa fornece uma discussão detalhada da etiologia, patogênese, avaliação e tratamento das olheiras infraorbitais. Uma compreensão da anatomia profunda e superficial é crucial para o manejo dessa entidade complexa. $\mathrm{O}$ arsenal para o tratamento inclui intervenções minimamente invasivas, como maquiagem $\mathrm{e}$ cosmecêuticos, uma variedade de tratamentos a laser e químicos, preenchimentos e transferência de gordura, bem como manobras cirúrgicas mais invasivas. 


\section{Resultados e Discussão}

A busca inicial dos dados resultou em 85 artigos, após aplicados os critérios de elegibilidade, foram incluídos nesta revisão 41 artigos.

As Olheiras afetam muitas pessoas, embora não sejam causa de preocupação médica, são esteticamente desagradáveis para muitos indivíduos, principalmente para mulheres, e podem ocorrer em todas as faixas etárias. Tem influência com relação a características hereditária, racial, tipo de pele, hábitos alimentares e de vida. A hiperpigmentação e a estase vascular das pálpebras foram consideradas como possíveis causas de olheiras, além de sombras acentuadas criado por reflexão da luz nos sulcos da área orbital (Mac-Mary et al., 2019; Park et al., 2018; Park et al., 2016).

Sua etiologia é complexa e multifatorial, e há vários fatores intrínsecos e extrínsecos associados à sua ocorrência (Gendler, 2005; Sarkar et al., 2016). Dentre os fatores intrínsecos, temos a genética, origem étnica, incluindo a quantidade e qualidade do pigmento melanina na pele, a estrutura óssea, grau de envelhecimento, alteração vascular, dificuldade drenagem, sinusite crônica que ocasiona um aspecto de olho fundo, mesmo em jovens, alterações hormonais, no período menstrual (Freitag \& Cestari, 2007; Hussain et al., 2019; Kontochristopoulos et al., 2016; Sheth et al., 2014; Vrcek et al., 2016).

A anatomia da região periorbital é complexa e também pode ser um fator contribuinte para formação das olheiras, já que a gordura subcutânea infraorbital é mínima e a área é propensa a estase de sangue e edema. Além disso, as pálpebras têm a pele mais fina do corpo humano, por transparência vemos o plexo vascular que é bem denso nessa região e até mesmo o próprio músculo orbicular. A estrutura óssea e o contorno da órbita podem causar efeitos de sombra ao redor do olho (MacMary et al., 2019; Park et al., 2018; Vrcek et al., 2016).

Em relação aos fatores extrínsecos, temos o estilo de vida, a exposição solar, atopias, alergia de contato, cansaço, fadiga, hiperpigmentação pós inflamatória (pós Operatório de cirurgia com depósitos de hemossiderina, processos inflamatórios que podem gerar acúmulo de líquido, com o tempo originam depósitos de pigmentos em processos crônicos), terapia hormonal, tabagismo, álcool. Certos medicamentos, como anti-inflamatórios não esteroides e quimioterápicos, também são desencadeantes de olheiras, os anticoncepcionais orais, análogos de prostaglandina; princípio ativo de colírio para tratamento de glaucoma que podem aumentar pigmentação da pele periocular (Freitag \& Cestari, 2007; Park et al., 2018; Park et al., 2016; Roh \& Chung, 2009; Sheth et al., 2014; Vrcek et al., 2016).

As olheiras podem ser classificadas em 5 tipos, de forma isolada ou combinada (Sawant \& Khan, 2020):

1: vascular

2: pigmentar

3: sulcos e sombras

4: por fatores exógenos

5: mista, por vários componentes (Sheth et al., 2014)

Uma outra classificação correlaciona os tipos de olheiras em relação aos fototipos de pele, segundo a classificação da escala de Fitzpatrick (Mac-Mary et al., 2019), podendo ser determinada da seguinte forma:

Fototipo 1 e 2: mais comuns, pele mais clara: por transparência há visualização dos vasos e o próprio músculo orbicular. Olheira vascular tem aspecto arroxeado, pele muito fina, que também pode ter relação hormonal e retenção líquido associados (Mac-Mary et al., 2019; Park et al., 2018).

Fototipo 3 e 4: Olheira pigmentar: melanocitose primária, exposição solar, gestação, melasma, pigmentação pós inflamatória, depósito de algum tipo de pigmento (Mac-Mary et al., 2019; Park et al., 2018).

Fototipo 5-6: mais relação com Olheiras pelos sulcos, depressões, envelhecimento, perda de volume médio facial, proeminência gordura orbital (Park et al., 2018; Park et al., 2016).

No processo de envelhecimento, há um remodelamento do arcabouço ósseo, a cavidade orbital se altera, aumentando 
diagonalmente, principalmente por perdas dos ossos zigomático e maxilar. E a têmpora, embora não leve a olheira, ajuda na queda da pálpebra, do tecido facial. Deixando o olho mais fundo e envelhecido (Jiang et al., 2016; Sawant \& Khan, 2020).

A estrutura ligamentar que faz as divisórias para os compartimentos de gordura na periórbita e terço médio da face, segura os coxins de gordura que também são perdidos nos compartimentos superficiais e profundos no processo envelhecimento. Alterações do relevo terço médio da face, gera sulcos e sombras, que podem evidenciar as bolsas de gordura das pálpebras e a bolsa malar (Lee \& Yen, 2017; Sawant \& Khan, 2020).

Importante avaliar a pálpebra inferior com terço médio face. Os tecidos ficam mais flácidos com excesso de pele, como se tivesse perdido o recheio. Tear trough (região medial até linha pupilar) e sulco pálpebro malar (região lateral a linha pupilar) são evidenciados (Hill et al., 2015; Jiang et al., 2016).

A região palpebral inferior pode ser subdividida em 3 regiões: Sulco Pálpebro Malar Lateral (SPML), Sulco Nasojugal (SNJ) e Deformidade Tear Trough (DTT), como demonstrado na Figura 1(Corduff, 2020; Couch \& Holds, 2013; Lee \& Hong, 2018).

Deformidade Tear Trough (DTT): o ligamento Tear Trough, representa a junção do ligamento Retentor orbicular e zigomático. O ligamento retentor orbicular fica entre as porções palpebral e orbital do músculo orbicular dos olhos. Quando se fala em deformidade Tear Trough, nos referimos ao afundamento da região e não ao ligamento (Lipp \& Weiss, 2019). Nesta região, há uma grande rede vascular, aderidos ao periósteo, estão as origens dos músculos elevadores do lábio superior e asa do nariz, além dos ligamentos propriamente ditos localizados de 3 a $4 \mathrm{~mm}$ do rebordo ósseo (Wong et al., 2012). Tendo em vista essas características anatômicas, a recomendação é aplicação imediatamente abaixo do orbicular dos olhos, evitando aprofundamento infraperiosteal pelo risco de lesão vascular e todas complicações relacionadas, preferencialmente com o uso de cânulas em microbolus pontuais bem distribuídos, com produto de reologia média (Lee \& Hong, 2018; Park et al., 2018).

Sulco Pálpebro Malar Lateral (SPML): corresponde a transição entre a pálpebra inferior e a região zigomática, formado pela deficiência ou deflação das estruturas da região, em especial os compartimentos de gordura (Wong et al., 2012). A aplicação de injetáveis nesta região deve ser preferencialmente com o uso de cânulas em microbolus bem distribuídos e massageados para melhor distribuição do produto de reologia média.

Sulco Nasojugal (SNJ): é a continuidade da Deformidade Tear Trough (DTT), vai do nariz à região jugal ou zigomática, podendo ser um leve esboço de sulco ou um sulco evidente. A aplicação de injetáveis no sulco deve ser feita em plano médio, abaixo do músculo orbicular, preferencialmente com o uso de cânulas em microbolus bem distribuídos com produto de reologia média (Bagci, 2018; Park et al., 2018). 
Figura 1. Regiões anatômicas da palpebral inferior.

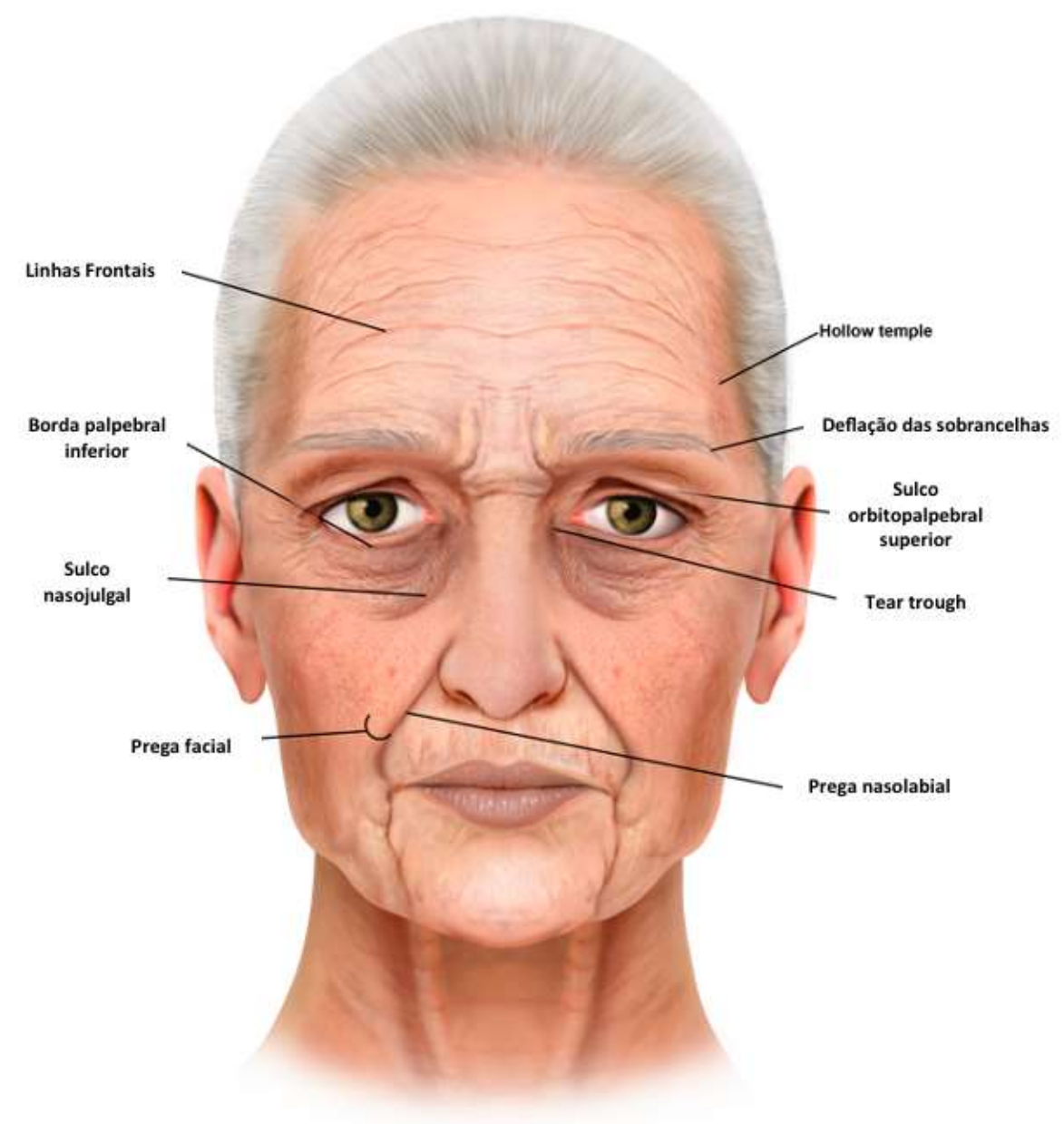

Fonte: Autores.

Um ponto a ser observado conjuntamente, quando se avalia preenchimento de olheiras, é avaliação da região malar, ou região zigomática, por ser considerada importante pilar estrutural da face, sendo muitas vezes a primeira região a ser abordada (Lee \& Yen, 2017; Park et al., 2018). Preferencialmente, deve-se abordar essa região, em plano supraperiosteal, com produto de reologia alta, em bolus sequenciais por retroinjeção com canula ou bolus com agulha, tentando mimetizar estruturas ósseas, semelhantes à marcação $\mathrm{Ck} 1$ e Ck2, da sistematização do MD Codes, podendo posteriormente realizar depósitos no subcutâneo profundo para amenizar qualquer irregularidade deixadas pelas projeções advindas dos bolus, em retroinjeção em leque (de Maio, 2020).

A região orbital lateral deve ser avaliada após os preenchimentos da região zigomática, superciliar e pálpebra inferior, não é incomum o afundamento dessa região. O preenchimento deve ser feito abaixo do músculo orbicular dos olhos, em retroinjeção linear, com produto de reologia média, para volumizar e também para efeito de miomodulação. Vale ressaltar que essa região contínua ao complexo zigomático e temporal, confere homogeneidade à essas unidades estéticas, fazendo uma projeção obliqua, margeando lateralmente os olhos, conferindo rejuvenescimento e beleza, abrindo o olhar e contribuindo com o "efeito lifting" (Jiang et al., 2016).

A região maxilar superior conhecida como região malar medial. Está em topografia do forame infraorbital e margeia a pálpebra inferior, dando suporte, de modo que o volume adequado dessa região, está associado a juventude e sensualidade. Assim quando bem estruturada, estabiliza, deixando as bolsas palpebrais e marcas ligamentares menos aparentes (Park et al., 2018). 
O preenchimento dessa região deve ser realizado em plano médio, na região das bolsas de gordura profundas malares que perdem seu volume com emagrecimento e envelhecimento, evitando o aprofundamento supraperiostal pelo risco de injeção intravascular e todas complicações catastróficas subsequentes. Preferencialmente com cânula, com produto de reologia média ou alta em retroinjeção em leque (Al-Shami, 2014).

Tratamento das olheiras depende do conhecimento sobre a etiologia e o seu tipo. Pois em diversas situações é necessário combinar terapêuticas e abordagens.

A seguir será abordado os principais métodos empregados para fins estéticos nestas regiões.

\section{Preenchedores}

Um dos segmentos de expansão mais rápida na indústria cosmética é a utilização preenchedores de ácido hialurônico (AH) e preenchedores não AH, tais como ácido poli-L-láctico e hidroxiapatita de cálcio. Em 2016, aproximadamente 2,5 milhões de procedimentos com preenchedores de HA foram realizados nos Estados Unidos. Isso foi 16,1\% maior do que em 2015 (Wong et al., 2012).

Os preenchedores não devem ser hidrofílicos devido à fina espessura da pele da região. Além disso devem ser injetados em microbolus, preservando a drenagem linfática existente nos pequenos septos que transitam em meio aos depósitos dos microbolus. Devem-se evitar retroinjeções lineares pois podem ficar visíveis pela pele translúcida e fina da região, formando uma deformidade "em cordão "inestética (Wong et al., 2012).

Os edemas intermitentes também são frequentes dada a fragilidade dos tecidos locais, de modo que pequenas retenções hídricas levam a congestão local, com bolsas palpebrais visíveis e prejuízo estético. Em virtude disto, recomendamse hipocorreções e correções seriadas para que no caso de retenção hídrica, não ocorra edema visível (Park et al., 2018).

Nestes casos, há preferência da maioria dos ácidos hialurônicos leves como Restylane $®$ vital, Belotero $®$ soft, Juvederm ${ }^{\circledR}$ volbella são recomendados para uso (Park et al., 2018).

Shah-Desay et al. em 2020, em uma série de casos intervencionais não randomizados, injetaram microbolus de peso molecular $(12 \mathrm{mg} / \mathrm{ml})$ de preenchedor de ácido hialurônico reticulado estabilizado (Restylane Vital Light TM) subdermicamente na pálpebra inferior e na zona pré-septal medial do Tear Through para voltar a volumizar e aumentar a espessura da pele das pálpebras. Os autores descreveram que baixos volumes, em média de $0,1 \mathrm{ml}$, reologia de baixo $\mathrm{G}$ prime, ou seja, baixo peso molecular com baixa higroscopia, técnica de injeção, usando agulhas estilo mesoterapia para que se coloque microgotículas de preenchedor subdermicamente, na localização limitada à parte superior 1/3 Tear Through superomedial e da cavidade préseptal superior, minimizando os riscos de complicações como edema palpebral de $1 \%$ em comparação com $11 \%$ relatado na literatura. Outros benefícios como a melhora relativa nas linhas e rugas sob os olhos, hidratação da pele e redução nas veias azuis visíveis (Shah-Desai \& Joganathan, 2020).

Shah-Desj (2020) desta que diversos estudos clínicos demonstraram que o tratamento com gel de pequenas partículas de ácido hialurônico não estabilizado por animais (NASHA) resulta em melhora da elasticidade da pele facial junto com melhora clínica na aparência da olheira, além do baixo risco vascular, pois as injeções são realizadas subdermicamente, e a vascularização está localizada no supra ou submuscular. No entanto, existe um alto risco de hematomas temporários (ShahDesai \& Joganathan, 2020).

\section{Plasma rico em plaquetas}

Outra opção de tratamento para olheiras é o plasma rico em plaquetas (PRP), fatores de crescimento que funciona como bioestimulador, promovem estímulo de colágeno (Al-Shami, 2014). Obtido através da centrifugação do sangue para remover os glóbulos vermelhos. Nesta técnica separa-se o plasma, que possui uma significativa concentração de fatores de 
crescimento, quimiocinas e citocinas e o utiliza para estimular a cura de vários processos. Recentemente, houve um aumento na aplicação de PRP nas áreas dermatológica e estética, principalmente para rugas, flacidez e olheiras secundárias. O PRP pode estimular a proliferação de fibroblastos dérmicos e a síntese de colágeno. O tratamento com PRP de patologias da área periorbital resulta em melhorias histológicas de fotoenvelhecimento, aumentos de pontuação de satisfação subjetiva dos pacientes e do avaliador da aparência da pele rejuvenescida (Evans et al., 2021).

O fator de crescimento transformador- $\beta 1$ e o fator de crescimento epidérmico presentes no PRP são sugeridos como responsáveis por inibir a produção de melanina via ativação de quinase regulada por sinal extracelular retardada e inibição da expressão de prostagandina-E2 / tirosinase. Além disso, o PRP melhora a sobrevivência do enxerto de gordura e pode ser usado em combinação com Lipoenxertia de gordura autóloga, técnica segura com baixo risco de rejeição, estimulando a elastina , stem cells, fatores de crescimento, melhorando a qualidade pele, para preenchimento de olheiras (Luo et al., 2020; Park et al., 2018).

\section{Hidroxiapatita de cálcio e Ácido poli-L-láctico}

Outros preenchimentos, como a hidroxiapatita de cálcio e ácido poli-L-láctico, que são considerados preenchedores semipermanentes (efeito que dura 12-24 meses), são amplamente utilizado para restauração de volume da face média, mas sua aplicação para correção da deformidade Tear Trough foi desencorajada devido à falta de precisão e aumento do risco de complicações (Montes, 2012).

No entanto, Corduff (2020) decreveu um novo tratamento para correção da pigmentação periorbital e/ou descoloração associada a DTT, em alguns casos, nos quais o AH apresentou resultados ruins, por realçar o excesso de pigmentação das olheiras. Propriedades reológicas e bioestimuladoras exclusivas da hidroxiapatita de cálcio e sua colocação em pequenas quantidades abaixo DTT, através de ligamento retentor orbicular, efetivamente elevou, sem adicionar volume em excesso (Corduff, 2020; Sawant \& Khan, 2020). Uma lavagem com hidroxiapatita de cácio hiperdiluida melhorou a espessura e aparência de descoloração da pele sobrejacente preseptal (Corduff, 2020).

A seleção do preenchedor determina a profundidade da injeção. Preenchedores semipermanentes ou de alta viscosidade, como ácido poli-L-láctico e hidroxiapatita de cálcio (Sculptra ${ }^{\circledR}$ e Radiesse ${ }^{\circledR}$ respectivamente), geralmente são injetados nos planos mais profundos em uma localização supra-periosteal, considerando que materiais "mais macios" ou menos viscosos podem ser injetado na localização mais superficial, subdérmica (Môle, 2018; Montes, 2012).

\section{Mesoterapia}

A Mesoterapia/biorevitalização com ácido hialurônico (AH) é uma abordagem de tratamento atualmente usada para o rejuvenescimento da pele. Vários produtos com uma ampla gama de formulações de policomponentes estão disponíveis no mercado. A maioria dessas formulações contém AH não reticulado em combinação com um coquetel de biorevitalização, formada por várias quantidades de vitaminas, minerais, aminoácidos, nucleotídeos, coenzimas e antioxidantes. Em estudo comparativo de técnicas de carboxiterapia, peeling químico e mesoterapia, A mesoterapia demonstrou melhoria mais significativa na pigmentação e o mais alto nível de satisfação do paciente (Ahmed et al., 2019; Môle, 2018; kui young Park, 2017).

\section{Carboxiterapia}

A carboxiterapia é uma técnica cosmética baseada na injeção subcutânea de dióxido de carbono que melhora a qualidade e a elasticidade da derme, aumentando a liberação de oxigênio para o tecido por meio de uma potencialização do efeito Bohr. Este procedimento estimula a produção de novos vasos sanguíneos, aumentando os níveis de fatores de 
crescimento. Este aumento do fluxo sanguíneo fornece oxigênio e nutrientes para a pele e vasos, o que acaba melhorando a aparência da pele (Ahmed et al., 2019; Kim et al., 2020).

\section{Fios de polidioxanoma (PDO)}

Fios PDO (polidioxanona) são utilizados para região infraorbital, em geral fio liso canulado, produzindo um bioestímulo local, melhorando a textura e flacidez da pele, através da indução de colágeno. A sua eficácia é baseada em múltiplas implantações (até várias dezenas de threads por lado) e repetidos (a cada 3-12 meses), de acordo com eixos paralelos retilíneos ou cruzados (Môle, 2018).

\section{Cosmecêuticos}

Uma variedade de tratamentos tópicos pode ser usada para tratamento com vários graus de sucesso. Dentre os Cosmecêuticos, as opções de tratamento tópico incluem agentes clareadores, despigmentantes: agem como antagonista de tirosinase ou alterando sua ação, destacam-se Hidroquinona, Ácido Kojico, Ácido retinóico, Cafeína e tretinoína e agentes despigmentantes como a albutina, um extrato da planta bearberry, corticosteróides, antioxidantes tópicos incluindo vitamina A, K, C e E, e ácido azelaico (Wong et al., 2012). O clareamento de áreas hiperpigmentadas pode ser obtido com um ou mais agentes tópicos. A maioria dos agentes tópicos pode ser eficaz, mas requer um longo período para alcançar bons resultados. Além do uso de longo prazo, esses agentes podem causar irritação ou prurido em alguns pacientes com pele sensível (Gendler, 2005; Kim et al., 2020; Park et al., 2018).

Entre os Tratamentos não invasivos: há os lasers, que são indicados para reduzir ou eliminar rugas e pigmentação, o que melhora o quadro geral e aparecimento de olheiras infraorbitais (Freitag \& Cestari, 2007).

\section{Laserterapia}

Vários lasers podem ser usados como tratamento, entre eles o Laser Q-switched, é seguro e eficaz para olheiras bem pigmentadas. Fotona 4D: laser de erbium+ ND:YAG , estimula o colágeno, a força muscular, para pigmento leve, melhorando a ruga. Os lasers Q-switch são um meio eficaz de tratar as olheiras, principalmente devido a alterações hiperpigmentares na pele. Dado o rápido tempo de relaxamento térmico dos melanossomos, os lasers Q-comutados com tecnologia de pulso de nanossegundos são ideais para tratar seletivamente os melanossomos enquanto causam trauma mínimo às estruturas circundantes. Os lasers de rubi comutado Q usam um comprimento de onda de $694 \mathrm{~nm}$, resultando na ruptura dos melanócitos, melanófagos e queratinócitos. Este comprimento de onda relativamente baixo é adequado para pacientes com pele de Fitzpatrick tipos I e II, mas a alta taxa de ruptura do melanossoma não é ideal para pacientes com pele mais pigmentada. Dois estudos separados demonstraram uma melhora maior que $40 \%$ nas olheiras pigmentadas após o tratamento com lasers de rubi Q-comutado. Retinóides adjuvantes ou neoadjuvantes e hidroquinona podem ser usados para melhorar o perfil de segurança e tratar quaisquer problemas de hiperpigmentação (Kounidas et al., 2020; Lipp \& Weiss, 2019).

Os lasers de alexandrita Q-switch apresentam um comprimento de onda de $755 \mathrm{~nm}$ de espectro médio, resultando em menos trauma aos melanossomas e maior segurança em tipos de pele de Fitzpatrick superiores. Os lasers Nd: Yag penetram profundamente na pele e têm efeito mínimo nos melanossomos, devido ao seu alto comprimento de onda (1063 nm). Isso permite maior segurança ao tratar indivíduos mais pigmentados, como a pele de Fitzpatrick tipo V e VI (Lipp \& Weiss, 2019; Vrcek et al., 2016).

Lasers fracionados e Radiofrequência ao melhorar qualidade da pele, são adequados para olheiras associadas a pele flácida e com rítides. A radiofrequência fracionada ou Fraxx com ou sem multiagulhas estimula produção de colágeno, melhora as rugas finas, causa retração da pele, melhorando a qualidade, entretanto pode pigmentar a pele, devendo ter cuidado 
com pacientes com melasma, mais seguro até fototipo 3. Os dispositivos de radiofrequência (RF) usam energia produzida por uma corrente elétrica (em oposição a uma fonte de luz como em um laser) para criar um efeito térmico que resulta na contração do colágeno e neocolagênese, minimizando os danos colaterais (Môle, 2018; Ruiz-Esparza, 2004).

A energia de RF não está sujeita à diminuição pela absorção ou dispersão do tecido e pode ser usada para tratar camadas de tecido profundo, como derme e gordura subcutânea, sem produzir queimaduras térmicas. Além disso, os dispositivos RF podem ser usados para tratar uma ampla variedade de tipos de pele, pois não usam energia à base de luz. Vrcek (2016) destacou que esses dispositivos produzem aumento do colágeno e que muitos autores descrevem como leve a moderado e mais sutil do que os efeitos observados com lasers ablativos ou fracionados; no entanto, a baixa morbidade inerente torna a RF uma escolha desejável se efeitos leves forem desejados com um perfil de baixo risco (Alster \& Lupton, 2007; RuizEsparza, 2004; Vrcek et al., 2016).

O Laser de $\mathrm{CO} 2$ fracionado é mais invasivo: Ideal para ser realizado em todo o rosto, incluindo pálpebras para não correr risco de alterações de pigmentação. O resurfacing a laser é um meio poderoso de tratar as olheiras de forma multimodal. Em primeiro lugar, as camadas superficiais da pele podem ser ablacionadas resultando na regeneração das camadas superficiais menos pigmentadas. Além disso, o conteúdo melhorado de colágeno na pele recapeada resulta em melhor camuflagem do orbicular subjacente e vascularização regional, que geralmente é responsável pelo aparecimento de olheiras. Os lasers de $\mathrm{CO} 2$ e granada de ítrio-alumínio com érbio (Er: YAG) são as tecnologias de laser não fracionado mais comumente usadas, tendo a água como alvo (Gendler, 2005; Vrcek et al., 2016).

A tecnologia de laser fracionado utiliza a mesma tecnologia dos lasers ablativos completos; no entanto, o resurfacing a laser fracionado reduz o dano térmico confluente de lasers ablativos completos para um padrão pixelado subtotal de ablação, deixando para trás a pele não tratada, o que permite menos tempo de inatividade e uma taxa de complicações menor. Os resultados do resurfacing com laser de $\mathrm{CO} 2$ fracionado mostraram ser comparáveis ao resurfacing ablativo tradicional; no entanto, com uma menor taxa de complicações e menos tempo de inatividade (Gendler, 2005; Vrcek et al., 2016).

\section{Luz intensa pulsada}

Outra opção é a Luz intensa pulsada (LIP), emitem luz no espectro visível e são aplicados à pele por meio de um gel de acoplamento. Uma lâmpada de alto rendimento emite um amplo comprimento de onda de luz na faixa de 500-1200 nm usando a corrente elétrica que passa por uma câmara cheia de xenônio. O cromóforo alvo dessa luz são os pigmentos dérmicos e epidérmicos na forma de hemoglobina e melanina; que resulta em fototermólise seletiva de áreas pigmentadas e/ou hipervasculares da pele. O resultado é uma tonalidade homogênea da pele. As configurações podem ser ajustadas para tratar alvos específicos de vários graus de pigmento; por exemplo, tratamento preferencial de hemoglobina em relação à melanina para o tratamento de áreas telangiectásicas. Os parâmetros de tratamento são individualizados e o LIP é ideal para pacientes com Fitzpatrick tipos I - II (Gendler, 2005; Kim et al., 2020; Lipp \& Weiss, 2019; Park et al., 2018; Roh \& Chung, 2009; Vrcek et al., 2016).

\section{Ultrassom microfocado}

Ultrassom microfocado constitui um sistema que usa dupla função; transdutores para fornecer energia de ultrassom microfocada em profundidades pré-selecionadas abaixo da superfície da pele ao mesmo tempo que fornece imagens de ultrassom de alta resolução das camadas da pele para garantir a precisão do fornecimento de energia. Absorção da energia do ultrassom causa vibração intermolecular e produção de calor a temperaturas em torno de $65^{\circ} \mathrm{C}$, causando desnaturação do colágeno e iniciando a síntese do colágeno, sem atingir a superfície da pele, criando zonas microcoagulativas previsíveis e reproduzíveis que iniciam uma resposta inflamatória concentrada. Visando a derme reticular profunda e o músculo superficial 
e os planos fasciais, como o sistema músculo-aponeurótico superficial, o platisma e a fáscia do músculo peitoral, esta tecnologia não ablativa aumenta a neocolagênese e a neoelastogênese de uma maneira nova, evitando muitas das complicações relacionadas ao aquecimento epidérmico observadas em vários outros dispositivos não ablativos (Wulkan et al., 2016).

Recebendo a aprovação da Drug Administration (FDA) em 2009 para levantamento não invasivo de sobrancelha e desde então tem demonstrado propriedades estimulantes do colágeno e melhorias na flacidez periocular (Barbarino, 2020).

Ao combinar as 2 modalidades ultrassom microfocado com o gel de ácido hialurônico de matriz polidensificada coesiva, observou-se grande eficácia em melhoria da aparência da área dos olhos em pacientes com flacidez da pele periocular e deformidades Tear Through, para o rejuvenescimento sinérgico de toda a área periocular (Barbarino, 2020).

\section{Blefaroplastia}

A Blefaroplastia inferior associada ou não ao sooflifting ou lifting do terço médio é um procedimento seguro e de baixo risco que produz excelentes resultados cosméticos em coxins adiposos infraorbitários, além do tratamento do excesso de pele e bolsas palpebrais, sulcos muito profundos, melhorando a bolsa malar ou a ressecção e transposição da gordura infraorbital transconjuntival quando não há excesso de pele associado (Anastassov \& St. Hilaire, 2006). O peeling químico adjuvante ou resurfacing a laser podem ser usados para tratar as causas pigmentares (Wong \& Mendelson, 2017).

Uma variedade de técnicas de blefaroplastia da pálpebra inferior estão disponíveis. A blefaroplastia por excisão de gordura transconjuntival é um meio eficaz de tratar o excesso de volume relativo superior ao ligamento orbitomalar que, quando combinado com a perda de volume na bochecha relacionada à idade, contribui para as olheiras. Quando as densas inserções do ligamento orbitomalar impedem que a blefaroplastia por excisão de gordura uniformize o contorno da pálpebrabochecha, a blefaroplastia com transposição de gordura da pálpebra inferior é um meio eficaz de tratar essas irregularidades de contorno. O ligamento retentor orbicular é liberado diretamente e a gordura orbital é transposta para a cavidade da borda infraorbital (Anastassov \& St. Hilaire, 2006; Wong \& Mendelson, 2017).

Pacientes com deformidades mais pronunciadas, orbitais graves com herniação de gordura e excesso de pele da pálpebra inferior costumam ser mais bem atendidos pela blefaroplastia inferior com reposicionamento de gordura ou combinação de lipoenxertia (Jiang et al., 2016).

O avanço do sistema orbicular e do sistema musculoaponeurótico superficial superior (SMAS) é um meio eficaz de suavizar ainda mais o contorno da pálpebra inferior e da bochecha. Em pacientes com festooning proeminente, que são dobras de pele sobre as bolsas malares, a elevação do SMAS pode ajudar na compressão dos festoons e na elevação do tecido mole malar para aumentar o volume da cavidade da borda infraorbital. Essas técnicas são particularmente úteis em situações onde a descida gravitacional desempenha um papel proeminente na etiologia das olheiras infraorbitais (Barbarino, 2020; Couch \& Holds, 2013; Kontochristopoulos et al., 2016; Park et al., 2018; Vrcek et al., 2016).

\section{Micro-agulhamento}

Outra técnica eficaz é dermaroller ou micro-agulhamento (MA) que envolve repetitivas punções da pele com microagulhas esterilizadas e gera trauma criando pequenos orifícios no extrato córneo e, assim, estimula a produção de colágeno e elastina e regenera a derme, na pálpebra recomenda-se agulhas de $0,5 \mathrm{~mm}$. Em um estudo relatado por Mohan $\mathrm{S}$ et al., 2020, um paciente com melanose periorbital estava sendo tratado com MA combinado com tratamento tópico de ácido kójico por 12 sessões (em intervalo de 2 semanas); com uma melhoria significativa na textura da pele e olheiras de $62,8 \%$. (Sawant \& Khan, 2020) 


\section{Peeling}

O peeling químico é definido como a aplicação de um ou mais agentes químicos que levam à destruição controlada da pele, resultando na remoção de lesões localizadas na epiderme ou na parte superior da derme. Este tratamento pode ser menos ou mais intenso de acordo com a intenção terapêutica e o tipo de peeling usado. Os peelings podem ser superficiais, médios ou profundos (Sawant \& Khan, 2020).

O peeling superficial com ácido tricloroacético (ATA) é ainda amplamente utilizado em concentrações de 15\%, 25\%, $50 \%$ e até $75 \%$, mais seguros o ATA a $10-20 \%$. Na maior concentração, há destruição da epiderme e derme superficial e a reepitelização ocorre a partir dos anexos epidérmicos (Gendler, 2005; Roh \& Chung, 2009).

Para o peeling médio, há os alfa-hidroxiácidos, que são um grupo de ácidos orgânicos amplamente encontrados em frutas e vegetais. Atuam reduzindo a coesão dos corneócitos por meio da inibição das enzimas envolvidas na formação de ligações iônicas, e estimulam a biossíntese de glicosaminoglicanos. O Ácido Glicólico é o alfa-hidroxiácido mais amplamente usado. Em concentrações de 50\% a 80\%, o ácido glicólico produz epidermólise, sendo neutralizado com bicarbonato de sódio a $1 \%$. O risco de efeitos colaterais e complicações, como despigmentação aumenta de acordo com a profundidade do agente de peeling químico e do fototipo de pele. Fototipos altos estão associados a uma maior suscetibilidade a hiperpigmentação e hipopigmentação pós-inflamatória. Portanto, candidatos adequados e os agentes químicos devem ser selecionados antes do procedimento. O Ácido Mandélico pode usar em todos fototipos (Gendler, 2005).

O Fenol, é um peeling profundo, que pode ser utilizado também associado de blefaroplastia transconjuntival.(Roh \& Chung, 2009) Por causa dos riscos de cicatrizes, hipopigmentação e toxicidade sistêmica, alguns autores não recomendam peelings de fenol (Gendler, 2005).

\section{Toxina Botulínica}

As rítides podem ser divididas no tipo estático (sempre presente) e o tipo dinâmico (apreciado na movimentação dos músculos). Rugas estáticas profundas são tratadas com procedimentos ablativos ou preenchimentos (Barbarino, 2020).

Rugas dinâmicas são tratadas de forma eficaz com neuromoduladores como a toxina botulínica. A Botulinum-A exotoxina (BTX-A) atua, bloqueando a contração muscular. BTX é uma neurotoxina isolado de Clostridium botulinum que impede a liberação de acetilcolina do neurônio pré-sináptico, causando temporariamente uma denervação química do músculo, levando à fraqueza muscular e paralisia. O BTX injetado na pálpebra inferior na orbita lateral, mostrou melhora nos famosos pés de galinha e aumento da abertura palpebral, devendo ser injetado subdermicamente $3 \mathrm{~mm}$ abaixo da margem ciliar até a linha médio pupilar. Em pacientes nos quais o músculo pré-tarsal ativo impacta o formato da borda infraorbital, o uso criterioso de Botox (Allergan Inc., Irvine, CA) pode diminuir a aparência das olheiras (Gendler, 2005; Môle, 2018).

\section{Outros tratamentos}

A fosfatidilcolina injetável pode ser outra opção para herniação de gordura infraorbital que pode beneficiar alguns pacientes com indicação de blefaroplastia, no entanto não é aprovada nos Estados Unidos para eliminação de gordura palpebral. Atualmente não há medicamentos injetáveis aprovados pela Food and Drug Administration (K. Y. Park et al., 2018).

O desoxicolato, apesar de seu uso não ser aprovado para nenhum procedimento estético realizado por nenhum profissional, no Brasil, ele ainda é amplamente utilizado off-label, especialmente corporal. Nos EUA e Canadá, é o único tratamento farmacológico aprovado para redução da gordura submentoniana (Jones et al., 2016).

Tatuagens e micropigmentação usam pigmento mais claro também podem ser utilizados para clarear olheira, uma ressalva importante é que a pele pode mudar com o tempo, se alterar com exposição solar. Camufla a olheira, não trata. A tatuagem na região infraorbital, também conhecida como blefaropigmentação (Angres, 1985; Roh \& Chung, 2009). 
Essa técnica utiliza uma agulha oca de ponta redonda que injeta pigmento na derme superficial. O pigmento inicialmente é depositado como grânulos livres na epiderme e derme, mas eventualmente migra para o tecido conjuntivo orbicular. Os grânulos de pigmento tornam-se endocitados por fibroblastos e permanecem no local permanentemente. A blefaropigmentação é comumente realizada para aumentar a margem palpebral inferior; no entanto, a tatuagem também tem sido usada para suavizar as transições na hiperpigmentação das pálpebras inferiores. Várias complicações foram relatadas; no entanto, a blefaropigmentação é tipicamente um procedimento bem tolerado (Vrcek et al., 2016).

Cuidados diários como qualidade de sono, praticar atividades relaxantes, beber mais água pois a hidratação melhora pele como um todo, uso de hidratantes faciais na área dos olhos, compressa geladas podem melhorar pela vasoconstrição local e massagem na região pode melhorar drenagem local. Em relação a alimentação, deve-se evitar o excesso de sal (Gendler, 2005; Vrcek et al., 2016).

Embora não seja tratamento, a maquiagem pode disfarçar as olheiras. Corretivos e cosmecêuticos são a opção menos invasiva para o tratamento das olheiras. A base de maquiagem mineral pode ser usada para misturar a tonalidade da pele e ocultar pigmentos mais escuros indesejáveis na região infraorbital. Além disso, usando técnicas opostas de paleta de cores, tons opostos podem ser usados para minimizar irregularidades de cores, de modo que, para olheiras de aspecto azul arroxeado recomenda-se o uso de corretivos em tons laranja. Já para aquelas de aspecto Verde acinzentada, em geral por acúmulo pigmento está mais indicado corretivos rosado (Gendler, 2005; Vrcek et al., 2016).

\section{Conclusão}

As etiologias do envelhecimento infraorbital são complexas e multifatoriais. Uma compreensão abrangente da anatomia e do processo de envelhecimento são necessários, de modo que diversas modalidades de tratamento são propostas, tanto em monoterapia quanto em combinação, para um efetivo rejuvenescimento dessa região. Cabe destacar que o objetivo do presente estudo foi realizar uma revisão ampla sobre as principais abordagens para a região palpebral inferior, sugerimos que demais pesquisadores realizem revisões sistemáticas da literatura sobre cada uma das intervenções descritas, pontuando as vantagens, desvantagens, indicações e complicações das respectivas técnicas.

\section{Referências}

Ahmed, N. A., Mohammed, S. S., \& Fatani, M. I. (2019). Treatment of periorbital dark circles: Comparative study of carboxy therapy vs chemical peeling vs mesotherapy. Journal of Cosmetic Dermatology, 18(1). https://doi.org/10.1111/jocd.12536

Al-Shami, S. (2014). Treatment of Periorbital Hyperpigmentation Using Platelet-Rich Plasma Injections. American Journal of Dermatology and Venereology, $3(5)$.

Alster, T. S., \& Lupton, J. R. (2007). Nonablative cutaneous remodeling using radiofrequency devices. Clinics in Dermatology, 25(5). https://doi.org/10.1016/j.clindermatol.2007.05.005

Anastassov, G. E., \& St. Hilaire, H. (2006). Periorbital and midfacial rejuvenation via blepharoplasty and sub-periosteal midface rhytidectomy. International Journal of Oral and Maxillofacial Surgery, 35(4). https://doi.org/10.1016/j.ijom.2005.09.013

Angres, G. G. (1985). The Angres Permalid-Liner method to enhance the result of cosmetic blepharoplasty. Annals of Ophthalmology, 17(3).

Bagci, B. (2018). A New Technique for the Correction of Tear Trough Deformity via Filler Injections. Plastic and Reconstructive Surgery - Global Open, 6(8). https://doi.org/10.1097/gox.0000000000001901

Barbarino, S. C. (2020). Combination Approach to Tear Trough Deformities and Under Eye Appearance Using Microfocused Ultrasound With Visualization and Hyaluronic Acid Filler. The American Journal of Cosmetic Surgery. https://doi.org/10.1177/0748806820947580

Corduff, N. (2020). An Alternative Periorbital Treatment Option Using Calcium Hydroxyapatite for Hyperpigmentation Associated with the Tear Trough Deformity. Plastic and Reconstructive Surgery - Global Open, 8(2), 1-7. https://doi.org/10.1097/GOX.0000000000002633

Couch, S. M., \& Holds, J. B. (2013). Lower eyelid blepharoplasty with fat repositioning. In Midfacial Rejuvenation. https://doi.org/10.1007/978-1-4614-10072_15 
de Maio, M. (2020). MD CodesTM: A Methodological Approach to Facial Aesthetic Treatment with Injectable Hyaluronic Acid Fillers. Aesthetic Plastic Surgery. https://doi.org/10.1007/s00266-020-01762-7

Evans, A. G., Ivanic, M. G., Botros, M. A., Pope, R. W., Halle, B. R., Glassman, G. E., Genova, R., \& Al Kassis, S. (2021). Rejuvenating the periorbital area using platelet-rich plasma: a systematic review and meta-analysis. Archives of Dermatological Research. https://doi.org/10.1007/s00403-020-02173-z

Freitag, F. M., \& Cestari, T. F. (2007). What causes dark circles under the eyes? In Journal of Cosmetic Dermatology (Vol. 6, Issue 3). https://doi.org/10.1111/j.1473-2165.2007.00324.x

Gendler, E. C. (2005). Treatment of periorbital hyperpigmentation. Aesthetic Surgery Journal, 25(6). https://doi.org/10.1016/j.asj.2005.09.018

Hill, R. H., Czyz, C. N., Kandapalli, S., Zhang-Nunes, S. X., Cahill, K. V., Wulc, A. E., \& Foster, J. A. (2015). Evolving Minimally Invasive Techniques for Tear Trough Enhancement. Ophthalmic Plastic and Reconstructive Surgery, 31(4). https://doi.org/10.1097/IOP.0000000000000325

Hussain, S. N., Mangal, S., \& Goodman, G. J. (2019). The Tick technique: A method to simplify and quantify treatment of the tear trough region. Journal of Cosmetic Dermatology, 18(6). https://doi.org/10.1111/jocd.13169

Jiang, J., Wang, X., Chen, R., Xia, X., Sun, S., \& Hu, K. (2016). Tear trough deformity: Different types of anatomy and treatment options. Postepy Dermatologii i Alergologii, 33(4). https://doi.org/10.5114/ada.2016.61607

Jones, D. H., Kenkel, J. M., Fagien, S., Glaser, D. A., Monheit, G. D., Stauffer, K., \& Sykes, J. M. (2016). Proper technique for administration of ATX-101 (Deoxycholic Acid Injection): Insights from an injection practicum and roundtable discussion. Dermatologic Surgery, 42. https://doi.org/10.1097/DSS.0000000000000875

Kim, J. S., Blizzard, S., Woodward, J. A., Leyngold, I. M., Liss, J., \& Freedman, S. F. (2020). Prostaglandin-Associated Periorbitopathy in Children and Young Adults with Glaucoma. Ophthalmology. Glaucoma, 3(4). https://doi.org/10.1016/j.ogla.2020.03.009

Kontochristopoulos, G., Kouris, A., Platsidaki, E., Markantoni, V., Gerodimou, M., \& Antoniou, C. (2016). Combination of microneedling and 10\% trichloroacetic acid peels in the management of infraorbital dark circles. Journal of Cosmetic and Laser Therapy, 18(5), https://doi.org/10.3109/14764172.2016.1157363

Kounidas, G., Kastora, S., \& Rajpara, S. (2020). Decoding infraorbital dark circles with lasers and fillers. Journal of Dermatological Treatment. https://doi.org/10.1080/09546634.2020.1855297

Lee, J. H., \& Hong, G. (2018). Definitions of groove and hollowness of the infraorbital region and clinical treatment using soft-tissue filler. In Archives of Plastic Surgery (Vol. 45, Issue 3). https://doi.org/10.5999/aps.2017.01193

Lee, S., \& Yen, M. T. (2017). Nonsurgical Rejuvenation of the Eyelids with Hyaluronic Acid Gel Injections. Seminars in Plastic Surgery, 31(1). https://doi.org/10.1055/s-0037-1598189

Lipp, M., \& Weiss, E. (2019). Nonsurgical Treatments for Infraorbital Rejuvenation: A Review. In Dermatologic Surgery. 45(5). https://doi.org/10.1097/DSS.0000000000001897

Luo, S., Zhang, X., Dong, H., Wen, C., \& Hao, L. (2020). Correction of the Tear Trough Deformity and Concomitant Infraorbital Hollows With Extracellular Matrix/Stromal Vascular Fraction Gel. Dermatologic Surgery: Official Publication for American Society for Dermatologic Surgery [et A1.], 46(12). https://doi.org/10.1097/DSS.0000000000002359

Mac-Mary, S., Zornoza Solinis, I., Predine, O., Jean-Marie, S., Sladen, C., Bell, M., \& O’Mahony, M. (2019). Identification of three key factors contributing to the aetiology of dark circles by clinical and instrumental assessments of the infraorbital region. Clinical, Cosmetic and Investigational Dermatology, 12. https://doi.org/10.2147/CCID.S217956

Môle, B. (2018). Técnicas adyuvantes de rejuvenecimiento facial. EMC - Cirugía Plástica Reparadora y Estética, 26(1). https://doi.org/10.1016/s16342143(18)89903-4

Montes, J. R. (2012). Volumetric considerations for lower eyelid and midface rejuvenation. In Current Opinion in Ophthalmology. 23,(5). https://doi.org/10.1097/ICU.0b013e3283560ab5

Park, Kui Young. (2017). Hyaluronic acid filler combining with antioxidants for infraorbital rejuvenation: Report of two cases. Journal of the American Academy of Dermatology, 76(6), AB159. https://doi.org/10.1016/j.jaad.2017.04.619

Park, K. Y., Kwon, H. J., Youn, C. S., Seo, S. J., \& Kim, M. N. (2018). Treatments of infra-orbital dark circles by various etiologies. In Annals of Dermatology. 30(5). https://doi.org/10.5021/ad.2018.30.5.522

Park, S. R., Kim, H. J., Park, H. K., Kim, J. Y., Kim, N. S., Byun, K. S., Moon, T. K., Byun, J. W., Moon, J. H., \& Choi, G. S. (2016). Classification by causes of dark circles and appropriate evaluation method of dark circles. Skin Research and Technology, 22(3). https://doi.org/10.1111/srt.12258

Pereira, A. S., Shitsuka, D. M., Parreira, F. J., \& Shitsuka, R. (2018). Método Qualitativo, Quantitativo ou Quali-Quanti. In Metodologia da Pesquisa Cientifica. https://repositorio.ufsm.br/bitstream/handle/1/15824/Lic_Computacao_Metodologia-Pesquisa-Cientifica.pdf?sequence=1.

Peng, P. H. L., \& Peng, J. H. (2018). Treating the tear trough: A new classification system, a 6-step evaluation procedure, hyaluronic acid injection algorithm, and treatment sequences. Journal of Cosmetic Dermatology, 17(3). https://doi.org/10.1111/jocd.12514

Roh, M. R., \& Chung, K. Y. (2009). Infraorbital dark circles: Definition, causes, and treatment options. In Dermatologic Surgery. 35(8). https://doi.org/10.1111/j.1524-4725.2009.01213.x

Ruiz-Esparza, J. (2004). Noninvasive Lower Eyelid Blepharoplasty: A New Technique Using Nonablative Radiofrequency on Periorbital Skin. Dermatologic Surgery, 30(2 I). https://doi.org/10.1111/j.1524-4725.2004.30052.x 
Research, Society and Development, v. 10, n. 5, e28710515033, 2021

(CC BY 4.0) | ISSN 2525-3409 | DOI: http://dx.doi.org/10.33448/rsd-v10i5.15033

Sarkar, R., Ranjan, R., Garg, S., Garg, V. K., Sonthalia, S., \& Bansal, S. (2016). Periorbital hyperpigmentation: A comprehensive review. Journal of Clinical and Aesthetic Dermatology, 9(1).

Sawant, O., \& Khan, T. (2020). Management of periorbital hyperpigmentation: An overview of nature-based agents and alternative approaches. In Dermatologic Therapy. 33(4). https://doi.org/10.1111/dth.13717

Shah-Desai, S., \& Joganathan, V. (2020). Novel technique of non-surgical rejuvenation of infraorbital dark circles. Journal of Cosmetic Dermatology. https://doi.org/10.1111/jocd.13705

Sheth, P., Shah, H., \& Dave, J. (2014). Periorbital hyperpigmentation: A study of its prevalence, common causative factors and its association with personal habits and other disorders. Indian Journal of Dermatology, 59(2). https://doi.org/10.4103/0019-5154.127675

Vrcek, I., Ozgur, O., \& Nakra, T. (2016). Infraorbital dark circles: A review of the pathogenesis, evaluation and treatment. Journal of Cutaneous and Aesthetic Surgery, 9(2). https://doi.org/10.4103/0974-2077.184046

Wong, C. H., Hsieh, M. K. H., \& Mendelson, B. (2012). The tear trough ligament: Anatomical basis for the tear trough deformity. Plastic and Reconstructive Surgery, 129(6). https://doi.org/10.1097/PRS.0b013e31824ecd77

Wong, C. H., \& Mendelson, B. (2017). Extended transconjunctival lower eyelid blepharoplasty with release of the tear trough ligament and fat redistribution. Plastic and Reconstructive Surgery, 140(2). https://doi.org/10.1097/PRS.0000000000003561

Wulkan, A. J., Fabi, S. G., \& Green, J. B. (2016). Microfocused Ultrasound for Facial Photorejuvenation: A Review. Facial Plastic Surgery, 32(3). https://doi.org/10.1055/s-0036-1584129 